\title{
Pyrofuse Modeling for EVTOL Aircraft DC Protection
}

\author{
Shadan Altouq, Kenny Fong, Patrick Norman, and Graeme Burt \\ University of Strathclyde
}

\begin{abstract}
Contemporary trends are leading towards the electrification of aircraft for urban mobility applications. Accordingly, there is a high demand for advancements in light-weight, high voltage technologies to realize these new aircraft types. Driven by recent developments in the automotive industry, hybrid Pyrofuse protection devices have emerged as one such new candidate technology. Pyrofuses offer rapid clearance of fault currents, reduced cost and weight when compared to conventional mechanical breakers. In addition, Pyrofuses have the ability to tune the time-current curve to fit the application's fault response characteristics. However, Pyrofuses are non-resettable devices whose exclusive use for electrical protection could present potential operational hazards and certification challenges in aerospace applications. Model-based analysis will be critical in supporting this evaluation. Accordingly, this paper offers the first complete design methodology to transiently model Pyrofuse operation in MATLAB/Simulink, drawing characteristics from commercially available datasheets. This model is then utilized to undertake an initial protection coordination feasibility study for a candidate eVTOL electrical system architecture, exploring the associated device and system level operational capabilities and limitations. In particular, the results show that the Pyrofuse can offer a good degree of nuisancetripping resilience against transient events whilst providing quick clearance of short circuit faults.
\end{abstract}

\section{Introduction}

Contemporary trends are leading towards the electrification of aircraft, with a recent particular focus on urban mobility applications. At present, key electrical technologies are sufficient for small eVTOL aircraft with a short range and low payload [1]. As electric aircraft platforms increase in size, greater levels of electric power generation and distribution are required, which in turn requires the use of higher operating voltages and DC power distribution to both reduce losses and be within designed weight margins. Future electric aircraft will likely require voltages up to, and in excess of 1000 VDC [2], whereby further development of associated key technologies is needed.

One of the key technology areas where there is considerable interest is in the development of solid-state protection devices. The associated typical fast fault interruption times, high power density and arc-less performance are desirable attributes for future aerospace applications $[3,4,5]$. However, the potentially short term Entry Into Service (EIS) targets for proposed eVTOL platforms mean that suitably rated devices may not be ready in time for use (solid state power controllers and circuit breakers are expected to be available on $\mathrm{N}+2$ and $\mathrm{N}+3$ timeframes respectively [7]). For example, initial eVTOL platforms are targeted to be in service by 2023 according to Uber [6]. As such, alternative solutions must be considered.
The automotive industry has recently introduced a hybrid Pyrofuse protection device as a solution to similar issues faced in the state of the art electric vehicles [8,9]. Pyrofuses have characteristics such as high power density, excellent protection sensitivity, good thermal cycling performance and the ability to tune time-current curves to fit system operating requirements [10]. There are two types of triggering mechanisms currently available for Pyrofuses, which are self-triggered and electronically triggered. The self-triggering method offers cost savings and less complexity than an electronic controlled Pyrofuse, as it eliminates the need for an external triggering device. However, selftriggered devices provide less functionality for tuning tripping characteristics [9].

In terms of current development, Panasonic and Gmbh [11] have presented a new type of Pyrofuse to provide fault protection and isolation for high power density battery applications. Bosch [12] and Texas Instruments [13] are developing current sensing circuits for externally triggered Pyrofuses. Concurrently Mersen [9] has also developed self-triggered Pyrofuses and electronically triggered Pyrofuses rated up to 1500 VDC. In addition, the authors in [9] have presented the testing of Mersen's self-triggered Xp series Pyrofuse with a fault level of $11 \mathrm{kA}$ at $500 \mathrm{VDC}$. The results presented show that the Pyrofuse successfully protected the circuit, interrupting the fault current at a maximum of $2 \mathrm{kA}$. Regarding aerospace applications, Mersen [9] in 2016 had also stated its intention to test the Xp-Pyrofuse series in an Airbus concept aircraft [10], although no publicly available update on this test has been provided to date.

Crucially, despite their other attractive qualities, Pyrofuses are nonresettable devices. The dependence on these to provide the primary power system protection functionality may present challenges for aerospace applications from a certification perspective [10]. Accordingly, with the lack of precedence of their operation in aerospace, better understanding of the Pyrofuses within eVTOL applications is required. This includes examining their performance under steady state electrical loading, faults and other transient conditions (e.g. as a result of load switching and lightning strike). Key to enabling this is the associated development of flexible and accurate circuit models. This paper is the first to present a methodology for modeling a complete self-triggered Pyrofuse in simulation software.

The paper first presents a review of Pyrofuse technology for higher voltage DC systems protection before presenting the modeling methodology for Pyrofuse circuit models. The use of the model for electrical system analysis is demonstrated in this paper through simulation-based case studies to highlight the model's sensitivity, selectivity and fast fault isolation. The modeling methodology utilizes parameters extracted from manufacturers' datasheets to allow for a more accurate representation of tripping profiles. Where there are gaps from the datasheets, assumptions and alternative calculation methods are provided.

Page 1 of 7 
Although Matlab/Simulink [14] was used for the implementation of the model by the author, the presented modeling methodology was designed to be generic and is suitable for a range of circuit simulation software packages.

\section{Model Formation}

\section{Pyrofuse Fundamentals}

The Pyrofuse design published in [9] was used as a reference for the model development. The self-triggering version of the Pyrofuse is modeled in this paper as this is the more complex version of two Pyrofuse options. The self-triggered Pyrofuse device consists of a pyroswitch (PS), two fuses $(\mathrm{F} 1, \mathrm{~F} 2)$ and a resistor ( $\left.\mathrm{R}_{\text {ignition }}\right)$ as shown in Figure 1. Similarly, the electronically triggered Pyrofuse can be easily derived from this model by replacing the sensor fuse (F1) with an alternative trigger signal in the model.

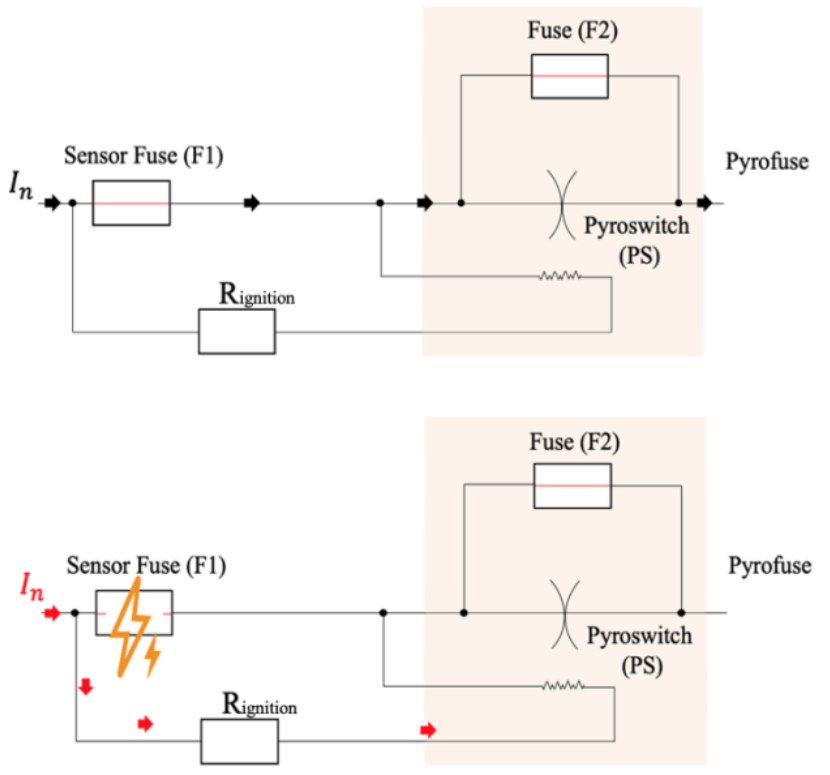

Figure 1. The operating conditions of Pyrofuse device

In hardware, the PS is placed on top of a copper conductor and utilizes a pyrotechnic trigger for separation/disconnection. The trip sequence starts with the F1 which is sized for the nominal circuit current flow and is underrated for voltage. F1 is in series with the conductor of the PS and allows nominal current flow. F1 operates when the initial accumulated fault current exceeds the trip threshold, which in turn causes the current to divert through the $\mathrm{R}_{\text {ignition. This diverted current }}$ causes the PS ignition which in turn separates the conductor. From this, the parallel fuse (F2) to the conductor is rated at nominal voltage, subsequently opens, extinguishing the inductive arcing that occurs as the conductor separates.

The sub model for F1 and F2 have the same model structure and operating sequence. This is further discussed in the following subsection.

\section{Fuse Sub-Model}

The fuse design published in $[15,16]$ is used as a reference for fuse elements F1 and F2 in the Pyrofuse circuit model. The flowchart in
Figure 2 summarizes the working sequence and control of the fuse model under short transient and permanent fault conditions. The component layout of the fuse model is illustrated in Figure 3. The fuse's switch state is 1 when it is closed $\left(F_{s}=1\right)$, and 0 when it is opened $\left(F_{s}=0\right)$.

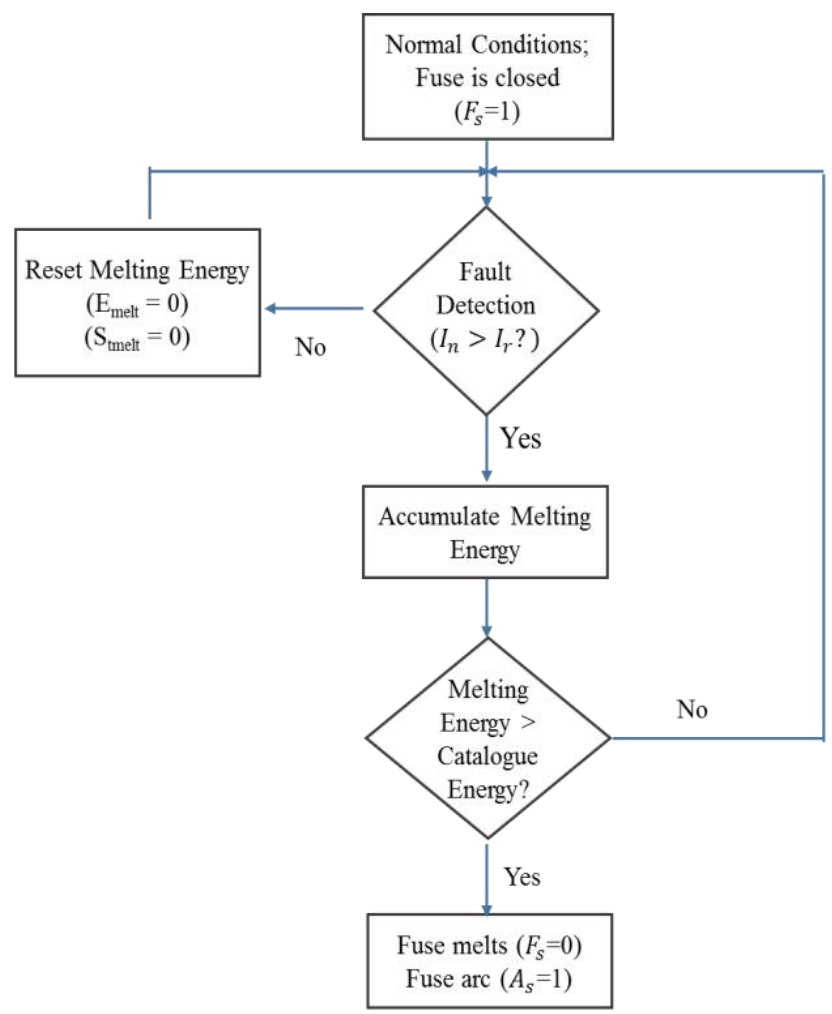

Figure 2. Flowchart of the Fuse Model Working Principle

Under normal conditions, the fuse switch is closed and the circuit current conducts through the fuse. If this current $\left(I_{n}\right)$ rises above the rated current threshold $\left(I_{r}\right)$ of the fuse, indicating the potential occurrence of a fault in the power system, the model will start to calculate the accumulated melting energy of the fuse $\left(E_{\text {melt }}\right)$. This calculation is performed over the period $\left(S_{\text {tmelt }}\right)$ from the fault occurrence $\left(t_{1}\right)$ to $\left(t_{2}\right)$, where $\left(t_{2}\right)$ is either the instance at which $\left(E_{m e l t}\right)$ is high enough to melt the fuse or the point at which $\left(I_{n}\right)$ becomes less than $\left(I_{r}\right)$ again, resetting the melting energy calculation to zero. This accumulated melting energy in $\mathrm{A}^{2} \mathrm{~s}$ is calculated using Equation (1).

$$
E_{\text {melt }}\left(\mathrm{A}^{2} \mathrm{~s}\right)=\int_{t_{2}}^{t_{1}} I_{n}^{2} t d t
$$

The catalog energy $\left(E_{c}\right)$ is the peak value of the melting energy of the fuse. If the calculated $\left(E_{\text {melt }}\right)$ is larger than the threshold $\left(E_{c}\right)$, the fuse will melt. The parameters to calculate $\left(E_{c}\right)$ are obtained from manufacturer's datasheet Time Current Curve (TCC). This can be converted to a regression function like that shown in Equation (2) within the sub model to dynamically calculate $\left(E_{c}\right)$ within the simulation. The variable $x$ in Equation (2) is the TCC melting time.

$$
\operatorname{LOG}\left(E_{c}\right)=a+b_{0}(x)^{1}+b_{1}(x)^{2}+b_{2}(x)^{3} \ldots
$$

When the fuse melts, the transition to arcing is modeled through the simultaneous opening of switch $\left(F_{S}\right)$ and closing of the arcing path switch $(A s)$ as shown in Figure 3. To emulate the arcing behavior of

Page 2 of 7 
the fuse, an RC equivalent circuit is utilized. This is highlighted in Figure 3.

There is limited information in the literature on specifying fuse arcing time, with most of the published papers acquiring the arcing time from either the clearing time in the manufacturer datasheet, experimental data or by utilizing the IEEE 1584 arcing time assumption [15,16,17]. The presented Pyrofuse model employs the IEEE assumption for the arcing time, and a method outlined in [16] whereby RC values are empirically tuned to provide a desired arcing time $\left(t_{a r c}\right)$ and peak current $\left(I_{0}\right)$.

Once the circuit current reaches zero, the arc is considered to be extinguished and the switch $\left(A_{S}\right)$ is opened again.

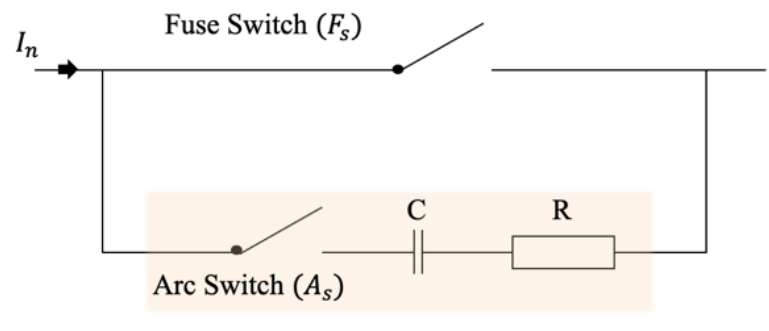

Arc Circuit

Figure 3. Equivalent Circuit of the Fuse when blown

\section{Pyroswitch Sub-Model}

The PS element within the Pyrofuse is an interrupter device that cuts into the conductor to isolate the fault. This disconnection is achieved by a small pyrotechnic charge actuating a miniature guillotine which cuts the conductor [9]. The flowchart in Figure 4 summarizes the control sequence of the PS model used to represent this disconnection under normal and fault conditions.

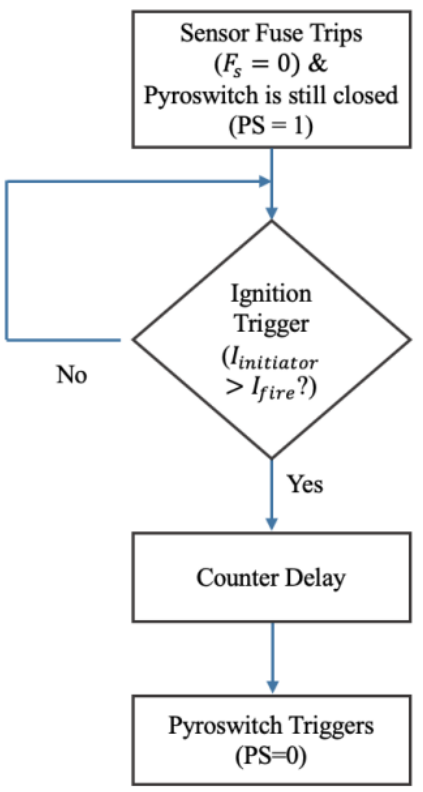

Figure 4. Flowchart of the Operating Conditions of Pyroswitch Device

Page 3 of 7
As shown previously in Figure 1, when the F1 operates, the current is forced to flow through the $R_{\text {ignition }}$ into the PS component. When the current in the resistive path (Initiator) exceeds the firing current threshold ( $\left.I_{\text {fire }}\right)$, the guillotine operation is activated. This action is emulated in the model by opening the associated switch element after a prescribed delay, representing the operating time of the guillotine (as specified on the product data sheets).

Under normal conditions F2 is in the closed state, but conducting minimal current (the PS element is considered to have a much lower impedance than F2). When the PS opens, current is diverted through F2, extinguishing any arc across the opened conductor of the PS.

\section{Fuse Sub-Model Parameterization and Testing}

This section describes the parameterization of the presented fuse submodel using manufacturer's datasheet extracts, and validates the operating time of the modeled device against datasheet specifications. A $1000 \mathrm{~V}, 800$ A FWJ fuse was chosen for this validation exercise [18].

The catalog energy for the FWJ-800 A fuse was extracted from the manufacturer's TCC [18], based on melting time and corresponding current. In the presented case, this was facilitated by utilizing a customized curve fitting function of the regression Equation (2) to derive a mathematical representation of the TCC as a function of time. This Equation represents the TCC curve for FWJ-800A from $5 \mathrm{kA}$ to $30 \mathrm{kA}$. Figure 5 shows the comparison of the data obtained from the TCC with customized curve fitting Equation.

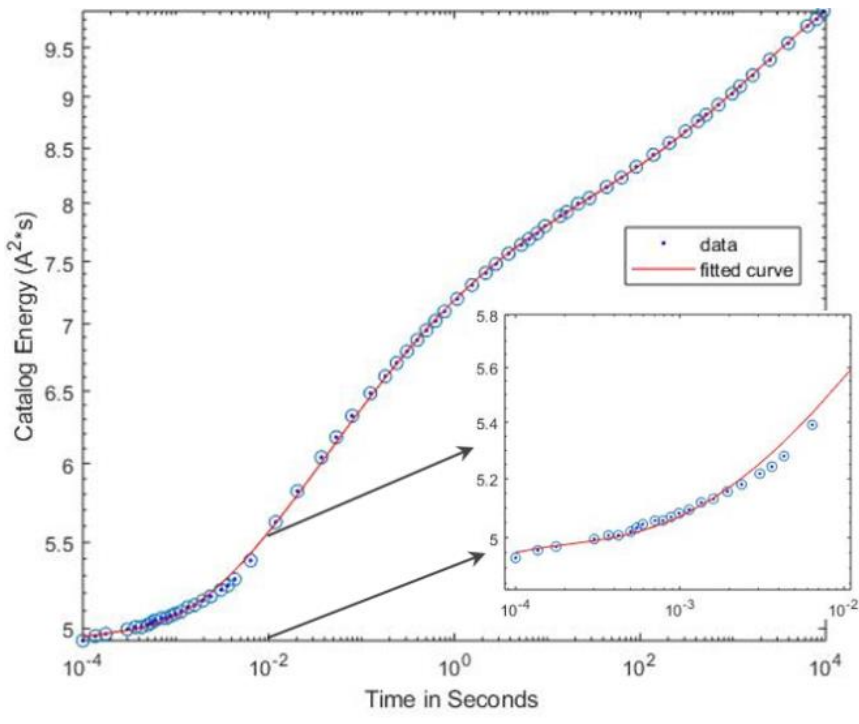

Figure 5. Curve-fitting the Catalog Energy data and the Melting Time extracted from Manufacturer's datasheet

To validate the modeled melting time, a range of different steady state fault current values were injected into the model and the resulting melting times were compared with associated values in the datasheet. Table 1 shows this comparison for fault currents ranging from $8000 \mathrm{~A}$ to $25,000 \mathrm{~A}$. It can be seen that the simulation results are typically within a $\pm 10 \%$ tolerance of the data acquired from the TCC in the manufacturer's datasheet. 
Table 1. Fuse Melting Time Difference between Simulation and Manufacturer's TCC

\begin{tabular}{cccc}
\hline $\begin{array}{c}\text { Fault Current } \\
(\mathbf{A})\end{array}$ & $\begin{array}{c}\text { Datasheet } \\
\text { Melting Time } \\
(\mathbf{s})\end{array}$ & $\begin{array}{c}\text { Simulation } \\
\text { Melting Time } \\
(\mathbf{s})\end{array}$ & Error $(\%)$ \\
\hline \hline 8000 & 0.00238 & 0.00249 & 5.04201681 \\
8500 & 0.001982 & 0.001985 & -0.1009082 \\
9000 & 0.0017 & 0.00164 & -2.3529412 \\
9500 & 0.00142 & 0.001396 & -2.1126761 \\
10000 & 0.00133 & 0.0012 & -9.7744361 \\
15000 & 0.00036 & 0.00034 & 8.33333333 \\
20000 & 0.00024 & 0.00023 & -4.1666667 \\
25000 & 0.000148 & 0.000144 & -4.0540541 \\
& & & \\
\hline
\end{tabular}

The arcing time can be deduced from the manufacturer's datasheet if the Total Clearing Time (TCC) is provided, as the arcing time is the difference between the TCC and the melting time. However, that is not the case for FWJ-800 A fuse datasheet, so assumptions were required to represent the arcing time in the model. IEEE-1584 specifies that the fuse's arcing time can be set to $10 \%$ of melting time plus an additional $0.004 \mathrm{~s} \mathrm{[17]}$. If the melting time for a specific fault is less than $0.01 \mathrm{~s}$, then $0.01 \mathrm{~s}$ is to be used for the arcing time [17].

Therefore, as the modeled fuse melting time was consistently less than $0.01 \mathrm{~s}$, the modeled RC values were set for an arcing time of $0.01 \mathrm{~s}$. For example, in case of a steady state fault of $20 \mathrm{kA}$ occurring at 0.01 $\mathrm{s}$, the fuse melts after a subsequent $0.00023 \mathrm{~s}$, and since the $10 \%$ of $0.00023 \mathrm{~s}$ is less than $0.01 \mathrm{~s}$, the modeled arcing time is thus set to 0.01 $\mathrm{s}$. The resulting total clearing time of the fault is $0.01023 \mathrm{~s}$. The arcing profile shown in Figure 6.

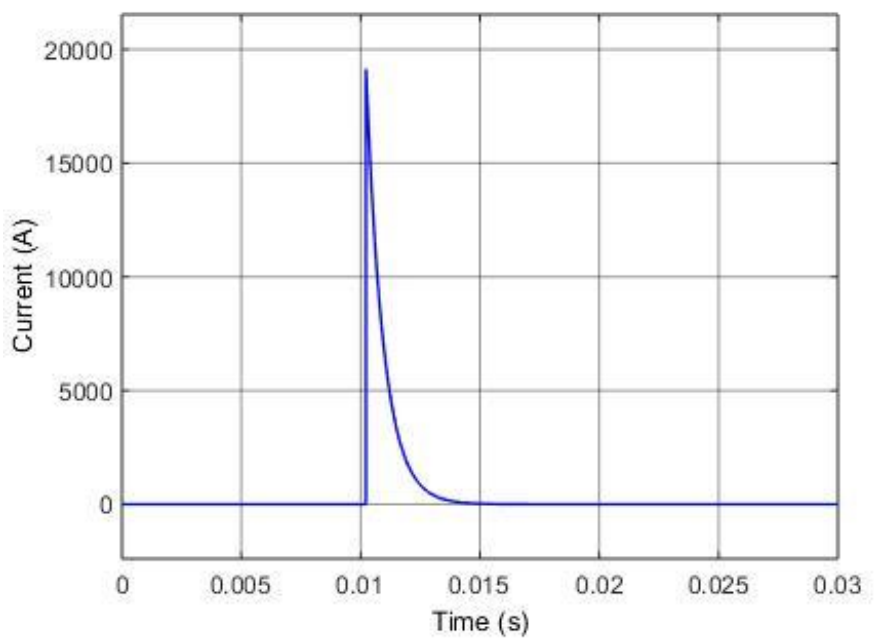

Figure 6. Arcing Current Profile through Arcing Path based on IEEE-1854 Assumption, using $R=1.77 \times 10^{-3} \Omega, C=20 \times 10^{-3} \mathrm{~F}$

Page 4 of 7
Although the IEEE-1584 specifications are sufficient for second-based time frame applications, future DC eVTOL and electric aircraft applications are likely to feature fault transients based on a millisecond time frame. As such, further study is required to derive a more accurate arcing time representation.

\section{Model Limitations}

Although the presented modeling method is comprehensive for performing an evaluation of Pyrofuse operation within an aircraft electrical system model, limitations of the model methodology exist and are presented below.

1. In case of intermittent faults, the fuse model does not account for the effect of the fuse's thermal cycling degradation after one fault peak.

2. When there is limited information in the manufacturer datasheet regarding the arcing time, assumptions or alternative calculations have to be made.

3. The model currently does not consider the ambient temperature of the fuse surroundings [16], nor the altitude impact on the Pyrofuse operating characteristics.

\section{Pyrofuse Simulation}

Using the presented modeling methodology, a representation of the Pyrofuse, as described in Figure 1, was modeled in Simulink, as shown in Figure 7. The following sections first show the model tested separately under different scenarios to illustrate the performance of the Pyrofuse model. Then a system level evaluation of multiple Pyrofuse models is performed using a simplified eVTOL power system architecture. The system level simulation demonstrates the tuning of multiple Pyrofuses to achieve protection coordination.

Table 2 shows the key parameters used for the power system model with an operating voltage of $600 \mathrm{VDC}$ and a nominal current flow of $400 \mathrm{~A}$. The datasheet used for obtaining the required parameters to model the fuses is given in [18].

Table 2. Key Parameter Values used in Simulation

\begin{tabular}{cc}
\hline Key Parameters & Value \\
\hline Circuit Voltage (Battery) & $600 \mathrm{~V}$ \\
Circuit Current & $\approx 400 \mathrm{~A}$ \\
Cable Resistance (R) & $0.1 \mathrm{~m} \Omega$ \\
Cable Inductance (L) & $0.01 \mu \mathrm{H}$ \\
DC Source Internal Resistance & $0.053 \Omega$ \\
DC Source Internal Capacitance & $250 \mathrm{nF}$ \\
\hline
\end{tabular}




\section{Pyrofuse Permanent and Transient faults Tests}

This section demonstrates the performance of the fuses and pyroswitch as a complete Pyrofuse model as shown in Figure 7. The current rating for the fuses in Figure 7 is $350 \mathrm{~A}$ for F1, and $70 \mathrm{~A}$ for the parallel F2. The model is tested by applying two transient self-cleared faults followed by a permanent fault as illustrated in Figure 8 . The shortcircuit faults are applied across the emulated motor load terminals as indicated in Figure 7. To test the sensitivity of the Pyrofuse model it was tested with different fault impedances. The fault is emulated using a snubberless ideal switch of $0.028 \Omega$ and $0.24 \Omega$, creating a transient current peak of $21.8 \mathrm{kA}$ and $2.7 \mathrm{kA}$ accordingly.

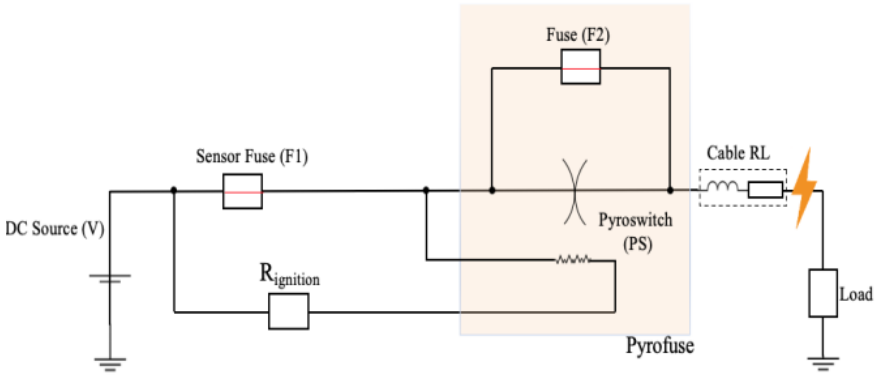

Figure 7. Complete Pyrofuse Model in Simulink

The first transient fault occurs at $0.01 \mathrm{~s}$ with a duration of $0.011 \mathrm{~ms}$, and the second at $0.015 \mathrm{~s}$ with a duration of $0.19 \mathrm{~ms}$. It can be seen in Figure 8 that the first two transient faults do not cause the melting energy in F1 to reach the catalog energy threshold. Thereby a reset signal was issued when the two transient faults were self-cleared, as indicated in Figure 8, resetting the melting time accumulation to zero. This restrains F1 from tripping the circuit. In practice, there may still be some thermal energy transiently retained in the fuse element, shortening the subsequent melting time.

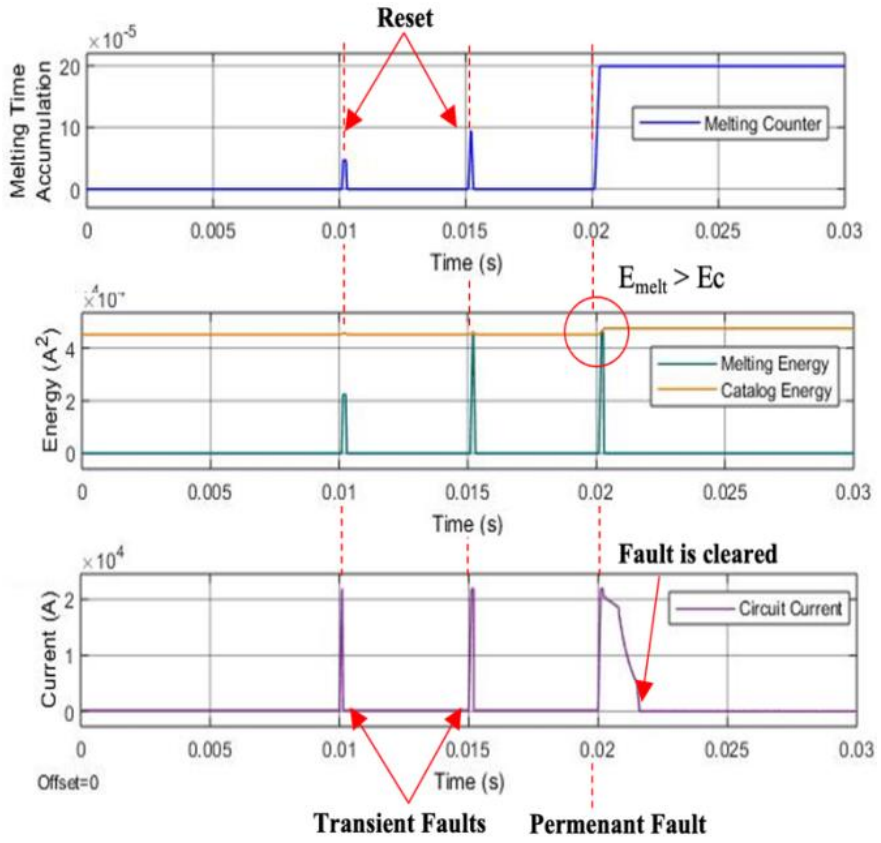

Figure 8. Performance Results of Sensor Fuse in the Pyrofuse Model under Short-duration and Permanent fault at 21.8. kA
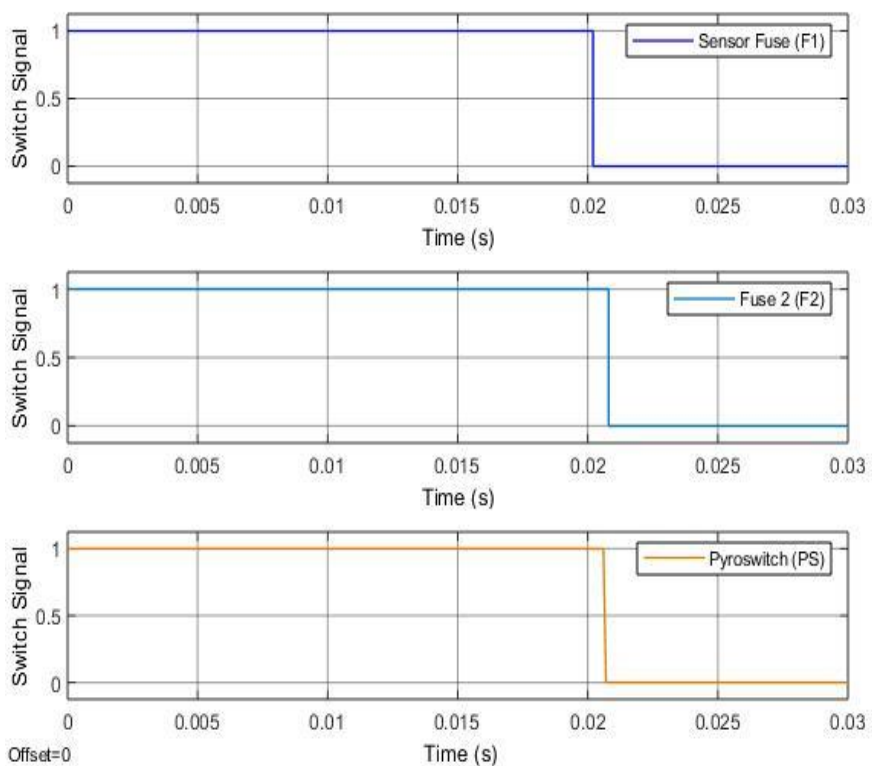

Figure 9 The Sequence of the Fuses and Pyroswitch tripping in the Pyrofuse Model for a fault of $21.8 \mathrm{kA}$

When the permanent fault occurs at $0.02 \mathrm{~s}$, the extended fault duration causes the melting energy to exceed the catalog energy and thus F1 trips at $0.02019 \mathrm{~s}(0.19 \mathrm{~ms}$ after fault occurrence). The current starts to flow into the resistive path. After passing a threshold specific to the PS, it is triggered and hence the circuit is cut at $0.0207 \mathrm{~s}(0.7 \mathrm{~ms}$ after fault occurrence). Immediately after the circuit is cut, the low rated current of F2 trips $0.1 \mathrm{~ms}$ after the PS has tripped, at $0.0208 \mathrm{~s}$, extinguishing the arcing from $\mathrm{F} 1$ and $\mathrm{F} 2$. The tripping sequencing of F1, PS and F2 is shown in Figure 9. Thus the total clearing time of the PS model is $1.6 \mathrm{~ms}$ for a $21.8 \mathrm{kA}$ low impedance fault.

The Pyrofuse model was also tested for a higher impedance fault, where a current of $2.7 \mathrm{kA}$ was injected in the model at $0.02 \mathrm{~s}$. With this fault type, F1 tripped at $0.4048 \mathrm{~s}$ after the fault occurrence, the PS then cut the circuit at $0.4063 \mathrm{~s}$ (1.5 ms after F1 tripping), and F2 tripped at $0.4064 \mathrm{~s}$. Following the extinction of the circuit arcing, the total fault clearing time was $0.432 \mathrm{~s}$.

Figures 8 and 9 show that the tripping sequence of the individual component models in the Pyrofuse model is working as required, and show the stability of the Pyrofuse under transient fault conditions. The coordination of multiple devices in a circuit is explored further in the following case study.

\section{Pyrofuse Coordination Validation}

A simplified circuit to represent a single branch in a generalized eVTOL aircraft power system architecture was modeled in Simulink, as shown in Figure 10. The generalized model is utilized to illustrate the protection grading and coordination of multiple Pyrofuse models for effective full-system protection. In this manner, only the Pyrofuse devices in the nearest upstream location of the fault should operate in order to preserve the security of supply to the loads where possible. The key parameters used in the circuit are given in Table 2 (shown earlier), while the ratings of the devices in the different Pyrofuse models are given in Table 3.

Page 5 of 7

$05 / 01 / 2021$ 
Permanent short-circuit faults were applied at different locations in the power system architecture, as indicated in Figure 10:

1) Across the emulated motor load terminals.

2) Between Pyrofuse $600 \mathrm{~A}$ and the DC Bus.

3) Between Pyrofuse $600 \mathrm{~A}$ and Pyrofuse $800 \mathrm{~A}$.

These faults were initiated at $0.02 \mathrm{~s}$ of simulation time and were realized using a snubberless ideal switch of $0.028 \Omega$, causing a theoretical transient peak in the circuit of $7.5 \mathrm{kA}$.

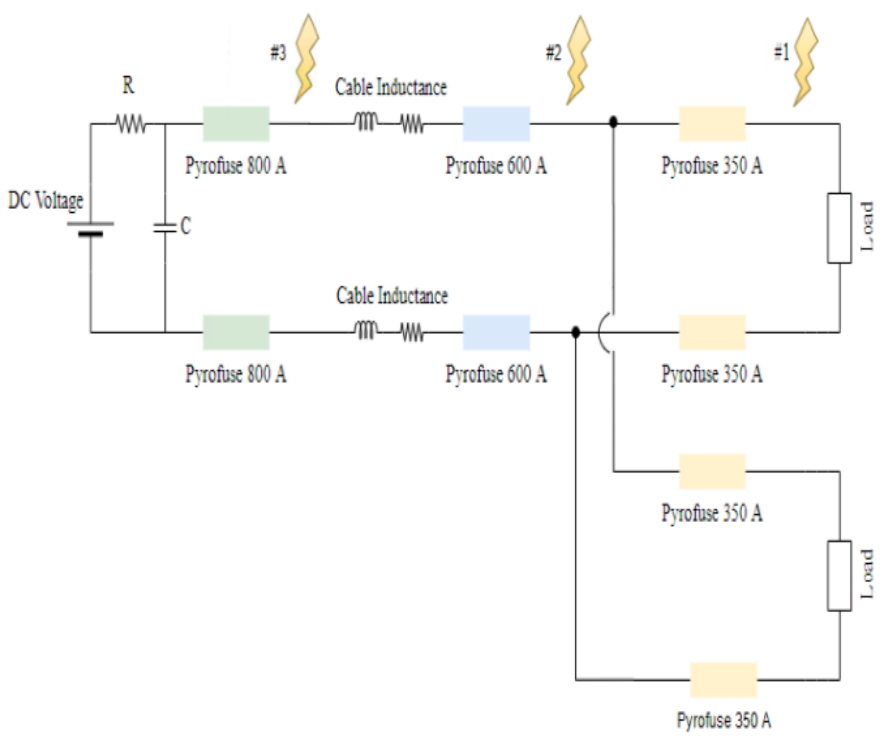

Figure 10. Simplified Model of a Single branch of eVTOL Power System Architecture

Table 3. Fuses rating selection for the Pyrofuses model in Figure 10

\begin{tabular}{ccc}
\hline Device Model & Sensor Fuse (F1) & Fuse (F2) \\
\hline \hline Pyrofuse-350 A & FWJ-350 A & FWP-70 A \\
Pyrofuse-600 A & FWJ-600 A & FWP-100 A \\
Pyrofuse-800 A & FWJ-800 A & FWP-150 A \\
\hline
\end{tabular}

Table 4 shows the operating times of various elements of the modeled Pyrofuse devices. For each of the fault locations, it can be seen that only the Pyrofuses nearest to the fault operate, enabling a continued supply of power to the healthy load for fault location 1 and to the upstream network buses for fault locations 2 and 3 respectively.
Table 4. Operating times of Pyrofuse elements for fault locations 1-3

\begin{tabular}{lccc}
\hline Fault location & Pyrofuse 350 & Pyrofuse 600 & Pyrofuse 800 \\
\hline \hline & F1 $=0.0214$ & & \\
Fault \#1 & PS $=0.0222$ & None & None \\
& F2 $=0.0223$ & & \\
& & F1 $=0.0238$ & \\
Fault \#2 & None & PS $=0.0247$ & \\
& & F2 $=0.0248$ & \\
Fault \#3 & & None & PS $=0.0256$ \\
& & & F2 $=0.0259$ \\
\hline
\end{tabular}

\section{Conclusions}

This paper offers the first published methodology for a complete circuit model of a Pyrofuse. This model enables the exploration of the design characteristics and operation of Pyrofuses within a DC system, and case studies are presented to showcase this. The Pyrofuse model has been shown to successfully remain stable during short-duration transient faults whilst clearing permanent low impedance faults in millisecond timescales, and shows sensitivity to high impedance faults. In addition, the tunability of the Pyrofuse device for use in a graded protection system has been demonstrated. This functionality, along with the potential for use of externally tripped devices, suggests that good protection coverage against a range of faults and failure modes can be realized with a purely Pyrofuse based protection system. However, as the Pyrofuse device is non-resettable, further exploration work is required to assess the potential compliance of a Pyrofuse-based protection system with regulatory rules before its consideration for use in an eVTOL or other aerospace applications. This is an area of further work for the authors, for which the presented pyrofuse model will provide a useful enabling capability for associated investigative simulations.

\section{References}

1. Roland Berger, "Aircraft Electrical Propulsion - Onwards and Upwards" Think:Act . London. 2018.

2. Jansen, R., Bowman, C., Jankovsky, A., Dyson, R., et al. "Overview of NASA Electrified Aircraft Propulsion (EAP) Research for Large Subsonic Transports" in 53rd AIAA/SAE/ASEE Joint Propulsion Conference, Atlanta, GA, 2017 doi: 10.2514/6.2017-4701.

3. R. Belchandan et al., "Characterization and Performance of $600 \mathrm{~V}$ 100A Solid-State Circuit Breaker", in 2018 9th IEEE International Symposium on Power Electronics for Distributed Generation Systems (PEDG), Charlotte, NC, 2018.

4. D. Molligoda, P. Chatterjee, C. Gajanayake, A. Gupta and K. Tseng, "Review of design and challenges of DC SSPC in More

Page 6 of 7 
Electric Aircraft", in 2016 IEEE 2nd Annual Southern Power Electronics Conference (SPEC), Auckland, New Zealand, 2016.

5. "ARPA-E awards $\$ 30 \mathrm{M}$ to 21 projects advance new class of highperformance power converters", Green Car Congress, 2019. [Online]. Available: https://www.greencarcongress.com/2017/08/arpa-e-awards30m-to-21-projects-advance-new-class-of-high-performancepower-converters.html. [Accessed: 27- Sep- 2019].

6. Uber, "The future of Urban Mobility". [online]. Available: https://www.uber.com/in/en/elevate/ [retrieved 1 Oct. 2020]

7. M. Flynn et al., "Protection and Fault Management Strategy Maps for Future Electrical Propulsion Aircraft", IEEE Transactions on Transportation Electrification, 2019. doi: 10.1109/tte.2019.2940882.

8. Koprivšek, M., "Advanced Solutions in Over-Current Protection of Hvdc Circuit of Battery-Powered Electric Vehicle", in PCIM Europe 2018, Nuremberg, Germany, 2018. ISBN: 978-3-80074646-0.

9. Tanaka, S., Ouaida, R., Chen, S., Chailloux, T., "Evaluation of Novel Hybrid Protection Based on Pyroswitch and Fuse Technologies", in The 2018 International Power Electronics Conference, Niigata, Japan, 2018. doi: 10.23919/IPEC.2018.8507793.

10. Mersen, "Current Limiting Device to Address DC Aeronautics Power Distribution Systems", 2013, [online]. Available: https://cordis.europa.eu/docs/results/641/641336/final1-finalreport.pdf_[retrieved $20 \mathrm{Mar} .2020]$

11. Lell, P., Volm, D., "Innovative Safety Concept to Shutdown Short Circuit Currents in Battery Systems up to $1000 \mathrm{~V}$ Based on Ultrafast Pyrofuse Technology", in IEEE Holm Conference on Electrical Contacts, Albuquerque, NM, 2018. doi: 10.1109/HOLM.2018.8611656

12. Bosch, "Explosions that save lives", [online]. Available: https://www.bosch-presse.de/pressportal/de/en/explosions-thatsave-lives-200641.html [retrieved 20 Mar. 2020]

13. Kambham, T. R. C. R., "PYRO-FUSE CIRCUIT", Taxas Instruments Incorporated, [online]. Available: http://www.freepatentsonline.com/y2019/0123542.html

14. MathWorks, "Simulink-Simulation and Model-Based Design", [online]. Available: https://uk.mathworks.com/products/simulink.html [retrieved 2 Oct. 2020]
15. Tian, W., Lei, C., Zhang, Y. et al, "Data analysis and optimal specification of fuse model for fault study in power systems", in IEEE Power and Energy Society General Meeting (PESGM), Boston, MA. 2016. doi: 10.1109/PESGM.2016.7741864

16. Li, D., Qi, L., "Energy based fuse modeling and simulation", in IEEE Electric Ship Technologies Symposium (ESTS), Arlington, VA, 2013. doi:10.1109/ESTS.2013.6523781.

17. IEEE Industry Applications Society. 2018. "IEEE Guide For Performing Arc-Flash Hazard Calculations", IEEE Std 15842002, Rev. 2018.

18. Cooper Bussmann, "High Speed Fuses", [online]. Available: http://www.cooperindustries.com/content/dam/public/bussmann/ Electrical/Resources/Catalogs/North_American_High_Speed_F uses.pdf [retrieved 20 Mar. 2020]

\section{Contact Information}

Shadan Altouq is within the Institute for Energy and Environment, Department of Electronics and Electrical Engineering, University of Strathclyde, Technology and Innovation Centre, 99 George Street, Glasgow, UK, G1 1RD. Email: Shadan.altouq@strath.ac.uk

\section{Acknowledgments}

This work was supported by the Rolls-Royce University Technology Centre program at the University of Strathclyde.

\section{Definitions/Abbreviations}

$\begin{array}{ll}\text { UAM } & \text { Urban Air Mobility } \\ \text { eVTOL } & \begin{array}{l}\text { Electrical Vertical Take-off } \\ \text { and Landing }\end{array} \\ \text { EIS } & \text { Entry Into Service }\end{array}$

